\title{
Asymmetric Spatial Frequency Tuning of Motion Mechanisms in Human Vision Revealed by Masking
}

\author{
Claire V. Hutchinson and Tim Ledgeway
}

Purpose. To investigate the spatial frequency selectivity of the human motion system by using the technique of visual masking.

Methods. Modulation-depth thresholds for identifying the direction of a sinusoidal test pattern were measured over a range of spatial frequencies $(0.25-4 \mathrm{cyc} / \mathrm{deg})$ in the absence and presence of a temporally jittering mask.

Results. At the lowest test frequency $(0.25 \mathrm{cyc} / \mathrm{deg})$, maximum masking occurred when the test and mask shared the same spatial frequency, decreasing as the difference in spatial frequency between the test and mask increased. However, as test spatial frequency increased, maximum masking began to shift to when the mask was presented at $\sim 1$ octave below the test spatial frequency. Control experiments demonstrated that the asymmetric masking functions at higher test spatial frequencies was not affected by mask amplitude nor was it an effect of speed. The results confirmed that the peak at 1 octave from the test still occurred when the potential for off-frequency looking was minimized by presenting two masks positioned equidistant in frequency from the test grating. Control experiments revealed, however, that the peak at 1 octave below the test was mediated by image size and/or the number of cycles presented on screen.

Concuusions. These findings provide support for the notion that motion perception is mediated by band-pass, spatial-frequency-selective mechanisms. Moreover, asymmetric tuning of the masking functions may reflect asymmetric spatial frequency selectivity of the mechanisms in the human visual system that encode motion or inhibition between mechanisms tuned to different spatial frequencies. (Invest Ophthalmol Vis Sci. 2007;48:3897-3904) DOI:10.1167/iovs.06-1056

$\mathrm{N}$ umerous psychophysical studies that have investigated visual processing at threshold contrast levels have shown that the visual system contains banks of independent, quasilinear, band-pass filters, each tuned to a particular range of spatial frequencies. ${ }^{1-10}$

Some of the most compelling evidence for the existence of spatial-frequency-selective mechanisms has come from masking studies. Masking effects are typically spatial frequency selective, occurring only when the test and mask patterns have similar spatial frequencies, and have been used to investigate the spatial frequency tuning of channels in the human visual

From the Visual Neuroscience Research Group, School of Psychology, University of Nottingham, Nottingham, United Kingdom.

Supported by a BBSRC (Biotechnology and Biological Sciences Research Council) Responsive Research Grant BB/C518181/1.

Submitted for publication September 6, 2006; revised January 30, April 3 and 23, 2007; accepted June 14, 2007.

Disclosure: C.V. Hutchinson, None; T. Ledgeway, None

The publication costs of this article were defrayed in part by page charge payment. This article must therefore be marked "advertisement" in accordance with 18 U.S.C. $\$ 1734$ solely to indicate this fact.

Corresponding author: Claire V. Hutchinson, Visual Neuroscience Research Group, School of Psychology, University of Nottingham, University Park, Nottingham NG7 2RD, UK;

lpxcvh@psychology.nottingham.ac.uk. system. $^{6,10-13}$ However, nearly all of these masking studies have been concerned with the characteristics of the channels that encode spatial form, and surprisingly few have used masking to examine directly whether spatial-frequency-selective channels exist for encoding motion. Anderson and Burr ${ }^{12}$ measured the spatial frequency, orientation, and temporal frequency selectivity of the visual motion system by using visual masking. As regards spatial frequency selectivity, masking was measured over a range of test spatial frequencies from 0.06 to $30 \mathrm{cyc} / \mathrm{deg}$, with and without a superimposed high contrast, temporally jittering mask. Masking was maximum when test and mask had the same spatial frequency and decreased as the spatial frequency difference between test and mask increased. Spatial frequency tuning functions were broadest (least selective) at low test spatial frequencies, becoming narrower (more spatial frequency selective) as test spatial frequency increased.

In a prior study, ${ }^{14}$ we investigated the spatial frequency selectivity of the mechanisms that encode second-order (contrast-defined) motion using the technique of visual masking. We measured spatial-frequency-selective effects over a range of test spatial frequencies $(0.125-2 \mathrm{cyc} / \mathrm{deg})$ in the presence of two counterphasing masks simultaneously superimposed above and below the test frequency. For second-order motion, masking functions were spatially band-pass and remained relatively invariant with changes in test spatial frequency. As a control, masking functions were also measured for first-order (luminance-defined) motion at a test spatial frequency of 0.5 cyc/deg. As expected, at a test frequency of $0.5 \mathrm{cyc} / \mathrm{deg}$, spatial frequency tuning was band-pass and resembled that found previously for luminance-defined motion patterns. ${ }^{12}$

We have since used the same masking technique (two counterphasing masks) to measure spatial-frequency-selective effects for first-order motion over an extended range of test spatial frequencies $(0.125-2 \mathrm{cyc} / \mathrm{deg})$. Of note, the results were unlike those found in previous studies in which firstorder motion stimuli were used ${ }^{12}$ or those found for secondorder motion in the experiment just outlined. ${ }^{14}$ At low test spatial frequencies $(0.125,0.25$, and $0.5 \mathrm{cyc} / \mathrm{deg})$ masking functions exhibited the expected band-pass character: masking (threshold elevation) was maximum when the test and mask shared the same frequency and decreased as the spatial frequency difference between the test and mask increased. However, at higher test spatial frequencies ( 1 and $2 \mathrm{cyc} / \mathrm{deg}$ ), masking functions showed two distinct peaks, one when test and mask were of the same spatial frequency and another when masks were presented at approximately \pm 1 octave from the test. Although this was an interesting preliminary finding, we were concerned that it may have been due to either (1) the type of masking paradigm that we had used (i.e., two counterphasing masks) or (2) the fact that we had used extremely low-contrast masks $(0.007 \%$ Michelson) to equate the visibilities of our first-order and second-order stimuli. Therefore, we decided to measure systematically masking functions for luminance-defined motion using the masking paradigm used previously by Anderson and Burr ${ }^{12}$ (one high-contrast, temporally jittering mask) to see wether the anomalous off-frequency peak observed at higher test spatial frequencies ( $\geq \sim 1-2 \mathrm{cyc} / \mathrm{deg}$ ) would persist under more conventional masking conditions. 
We measured spatial-frequency-selective masking by using test spatial frequencies of $0.25,0.5,1,2$, and $4 \mathrm{cyc} / \mathrm{deg}$. The procedure used differed slightly from that used by Anderson and Burr. ${ }^{12}$ They kept five cycles of test grating on-screen in all conditions and varied viewing distance, and consequently image size, to change spatial frequency. This meant that the degree to which the stimuli encroached on peripheral regions of the retina varied inversely with spatial frequency. For example, a test grating of $0.06 \mathrm{cyc} / \mathrm{deg}$ subtended more than $83^{\circ}$ at the retina whereas a grating of $30 \mathrm{cyc} / \mathrm{deg}$ was confined to a central region subtending less than $0.17^{\circ}$. Because different regions of the retina can differ greatly in their spatiotemporal sensitivity to motion, ${ }^{15}$ it remains to be seen whether such large variations in the spatial extent of the test gratings has a profound influence on the shapes of the masking functions measured at different spatial frequencies. In the present study, viewing distance remained constant, and the number of cycles of test grating varied from 5 cycles at a test spatial frequency of $0.25 \mathrm{cyc} / \mathrm{deg}$ to 80 cycles at $4 \mathrm{cyc} / \mathrm{deg}$.

\section{Materials ANd Methods}

\section{Observers}

Four observers took part in the study. The authors served as observers in all experiments. Observers MAB and BSW (naive observers) were used to confirm the generality of key findings. All observers had normal (BSW) or corrected-to-normal (CVH, TL, MAB) visual acuity and had no history of any visual disorders. All experimental procedures adhered to the tenets of the Declaration of Helsinki. Each observer's gender, age, refractive error, and visual acuity are given in Table 1.

\section{Apparatus and Stimuli}

Test stimuli were generated on computer (Macintosh G4; Apple Computer, Cupertino, CA) and presented on a monitor (Trinitron; Sony, Tokyo, Japan) with an update rate of $75 \mathrm{~Hz}$ with custom software written in the $\mathrm{C}$ programming language. For precise control of luminance contrast, the number of intensity levels available was increased from 8 to 14 bits by combining the outputs of the three digital-toanalog converters of the video card using a Bits ++ video attenuator (Cambridge Research Systems; Cambridge, UK). The mean luminance of the display was $55.25 \mathrm{~cd} / \mathrm{m}^{2}$. Images were viewed binocularly and in darkness at a distance of $73 \mathrm{~cm}$. One pixel subtended 1.56 arc min of visual angle resulting in a display region that subtended $20^{\circ}$ horizontally and vertically.

Stimuli were horizontally oriented, luminance-defined gratings $(0.25,0.5,1,2$, or $4 \mathrm{cyc} / \mathrm{deg})$ drifting at a temporal frequency of $5 \mathrm{~Hz}$. The initial absolute (starting) phase of the test stimulus was randomized on each presentation, and the direction of drift could be either up or down. Modulation-depth (contrast) thresholds were measured for test gratings in both the absence (baseline thresholds) and presence of a horizontally oriented, spatially sinusoidal mask that was positioned randomly from frame to frame so that it appeared to jitter with no net direction of motion. ${ }^{12}$ The mask was temporally broadband and had a Michelson contrast of 0.25 . Mask patterns were superimposed on the test grating at various spatial frequency intervals ranging from 3 octaves (two spatial frequencies that differ by 1 octave have a ratio of $2: 1$ ) below to 3 octaves above the test frequency.

The total duration of each stimulus presentation was $853 \mathrm{~ms}$, and the overall modulation depth of the pattern was smoothed on and off by a half cycle of a raised cosine lasting $170 \mathrm{~ms}$. In a similar manner, each stimulus was spatially windowed in the vertical dimension according to a half cycle of a raised cosine function with a half period of $4^{\circ}$. This procedure was used to minimize the presence of temporal and spatial transients.

\section{Procedure}

A single-interval, two alternative, forced-choice procedure was used. On each trial, observers were presented with a fixation cross followed by the presentation of the stimulus. After the presentation of the stimulus, observers were cued to respond with a key press, and their task was to judge the direction of the test grating's motion (up or down). The modulation depth of the test stimulus was varied from trial to trial, according to a modified 1-up 3-down staircase designed to converge on the modulation-depth corresponding to a $79.4 \%$ correct performance. ${ }^{16,17}$ At the beginning of each run of trials, the modulation depth of the test grating was initially set to a suprathreshold level ( $\sim 10 \mathrm{~dB}^{2}$ above threshold; in this case, $\mathrm{dB}$ is defined as: $20 \log _{10}\left[c / c_{t}\right]$, where $c$ is suprathreshold test contrast and $c_{t}$ is test contrast at threshold) and the initial staircase step size was chosen to be half this value. On subsequent reversals the step size was halved (with the constraint that it could not have a Michelson contrast of $<0.001$ ), and testing was terminated after a total of 16 reversals. Threshold estimates were taken as the mean of the last four reversals in each staircase. Each observer completed a minimum of four runs of trials (i.e., four staircases) for each condition and the order of testing was randomized. The mean threshold and the SEM were then calculated for each condition.

\section{Results}

\section{Experiment 1: Spatial Frequency Selectivity of the Human Motion System}

We began by measuring spatial-frequency-selective masking at test spatial frequencies of $0.25,0.5,1,2$, and $4 \mathrm{cyc} / \mathrm{deg}$. The masking functions obtained at each test spatial frequency are shown in Figure 1. In agreement with previous findings, ${ }^{12}$ masking functions were spatially band-pass and were broadest at the lowest test frequency $(0.25 \mathrm{cyc} / \mathrm{deg})$, becoming more narrowly tuned as test spatial frequency increased. At a test frequency of $0.25 \mathrm{cyc} / \mathrm{deg}$ maximum masking occurred when the test and mask shared the same frequency and the magnitude of the masking effect decreased as the spatial frequency difference between the test and mask increased. However, as test spatial frequency increased, maximum masking began to shift to mask spatial frequencies $\sim 1$ octave below the test.

Table 1. Gender, Age, Refractive Error and Visual Acuity for Each Observer

\begin{tabular}{lll}
\hline \multicolumn{1}{c}{ Observer } & \multicolumn{1}{c}{ Refractive Error } & \multicolumn{1}{c}{ Visual Acuity (logMAR)* } \\
\hline CVH (female, 26 y) & RE: -1.25 DS & RE: -0.10 \\
TL (male, 37 y) & LE: -0.75 DS & LE: -0.12 \\
& RE: -0.50 DS & RE: 0 \\
MAB (male, 27 y) & LE: plano & LE: 0 \\
BSW (male, 35 y) & RE: -2.00 DS/-0.50 @ 180 DC & RE: -0.08 \\
& LE: -2.00 DS/ -0.25 @ 170 DC & LE: -0.10 \\
& RE: +0.25 DS & RE: 0 \\
& LE: plano & LE: -0.04 \\
\hline
\end{tabular}

* Bailey-Lovie chart. 
Figure 1. Masking functions measured for test patterns with spatial frequencies of $0.25,0.5,1,2$, or 4 cyc/deg for observers CVH and TL, and 0.25 or $4 \mathrm{cyc} / \mathrm{deg}$ for observers $\mathrm{MAB}$ and BSW. Each plot indicates the degree of masking (filled symbols) at each test-mask frequency difference (in octaves). Plots include unmasked (baseline) thresholds (open symbols) for each spatial frequency tested. The Michelson contrast of each mask was 0.25 . At a test spatial frequency of $4 \mathrm{cyc} / \mathrm{deg}$, masking was measured only up to 2 octaves above the test frequency because, beyond this, the number of cycles required to represent the masks approached the Nyquist limit of the display. Error bars, \pm 1 SEM.
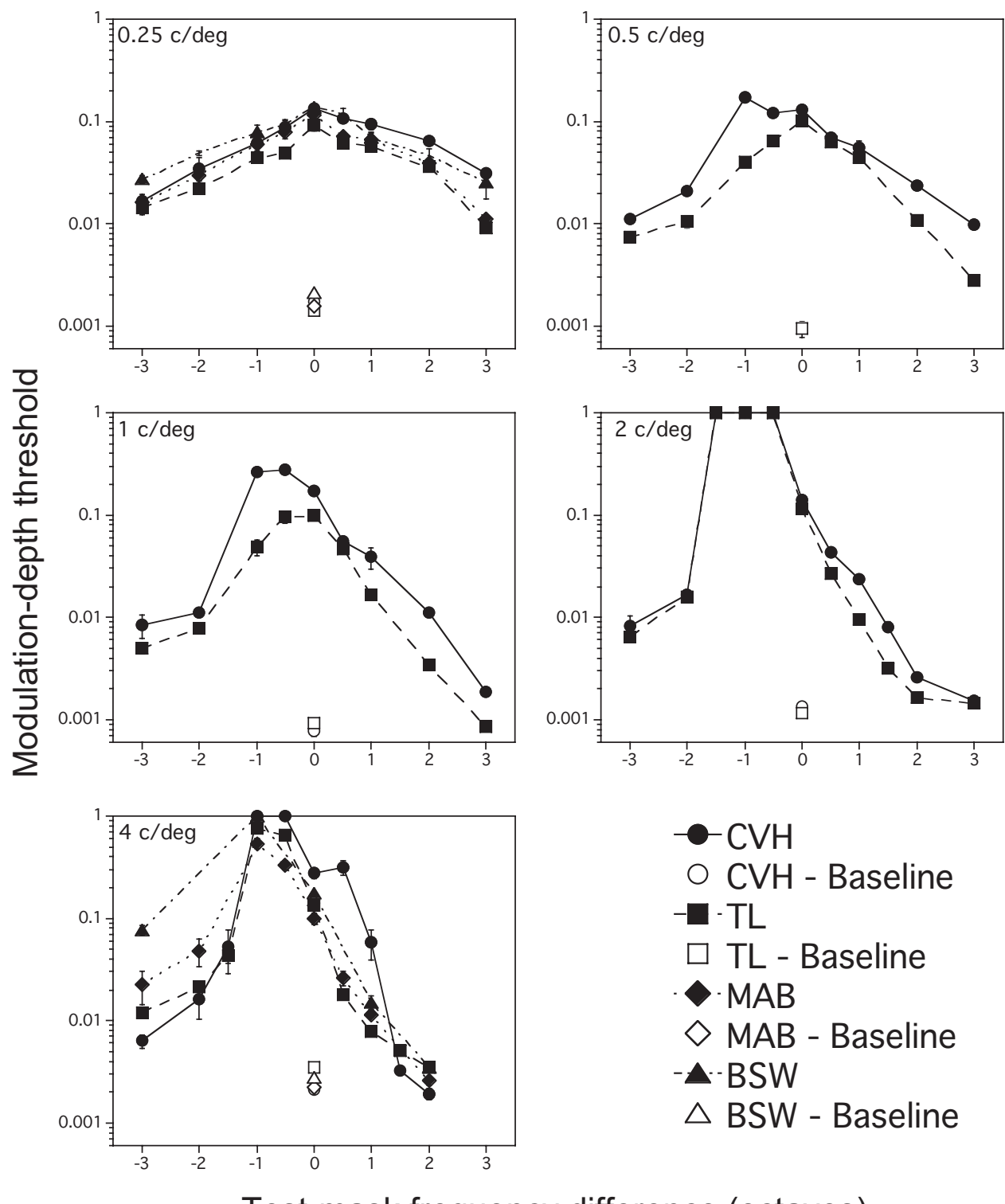

Test-mask frequency difference (octaves)

Indeed at test frequencies of 2 and $4 \mathrm{cyc} / \mathrm{deg}$, in some instances masking effects were so large that a threshold could not be reliably measured. When this was the case, modulation depth thresholds have been nominally plotted as 1 . In addition, masks below the test frequency elicited a greater level of masking than those above the test frequency.

\section{Experiment 2: The Effects of Mask Modulation Depth}

It is clear from Figure 1 that at higher test spatial frequencies, masking functions were band-pass in character but severely asymmetric (peak at $\sim 1$ octave below the test). In an attempt to rule out the possibility that the asymmetry could be in some way due to the high contrast of the masks, masking functions was remeasured at $2 \mathrm{cyc} / \mathrm{deg}$, with mask contrasts of 0.125 , 0.0625 , and 0.03125 (Michelson). The results are presented in Figure 2 and make it clear that the amount of masking scaled (became less) almost perfectly with decreasing mask contrast. However, maximum threshold elevation still occurred when the mask was positioned $\sim 1$ octave below the test spatial frequency, irrespective of the contrast of the jittering mask pattern.

Another possibility was that the shape of the masking functions (i.e., the peak off-test frequency) may reflect underlying sensitivity to the masks themselves. For example, if all mask patterns were presented at say a contrast of 0.25 , the mask spatial frequency to which observers were most sensitive would be of higher effective contrast to the visual system than the mask spatial frequency to which observers were least sensitive. Therefore, the greatest degree of masking would occur at the mask frequency to which observers were most sensitive. To investigate, we measured detection thresholds for each mask using a two-interval, forced-choice procedure. A jittering grating appeared in one of the two intervals, and the observer's task was to judge in which interval it had appeared. Thresholds were estimated by using an adaptive staircase technique in a manner analogous to that described in the Methods section. Modulation sensitivity functions (the reciprocal of modulation depth at threshold) for the masks are shown in Figure $3 \mathrm{a}$. The shape of the modulation sensitivity functions suggests that the shape of the masking functions, especially at 

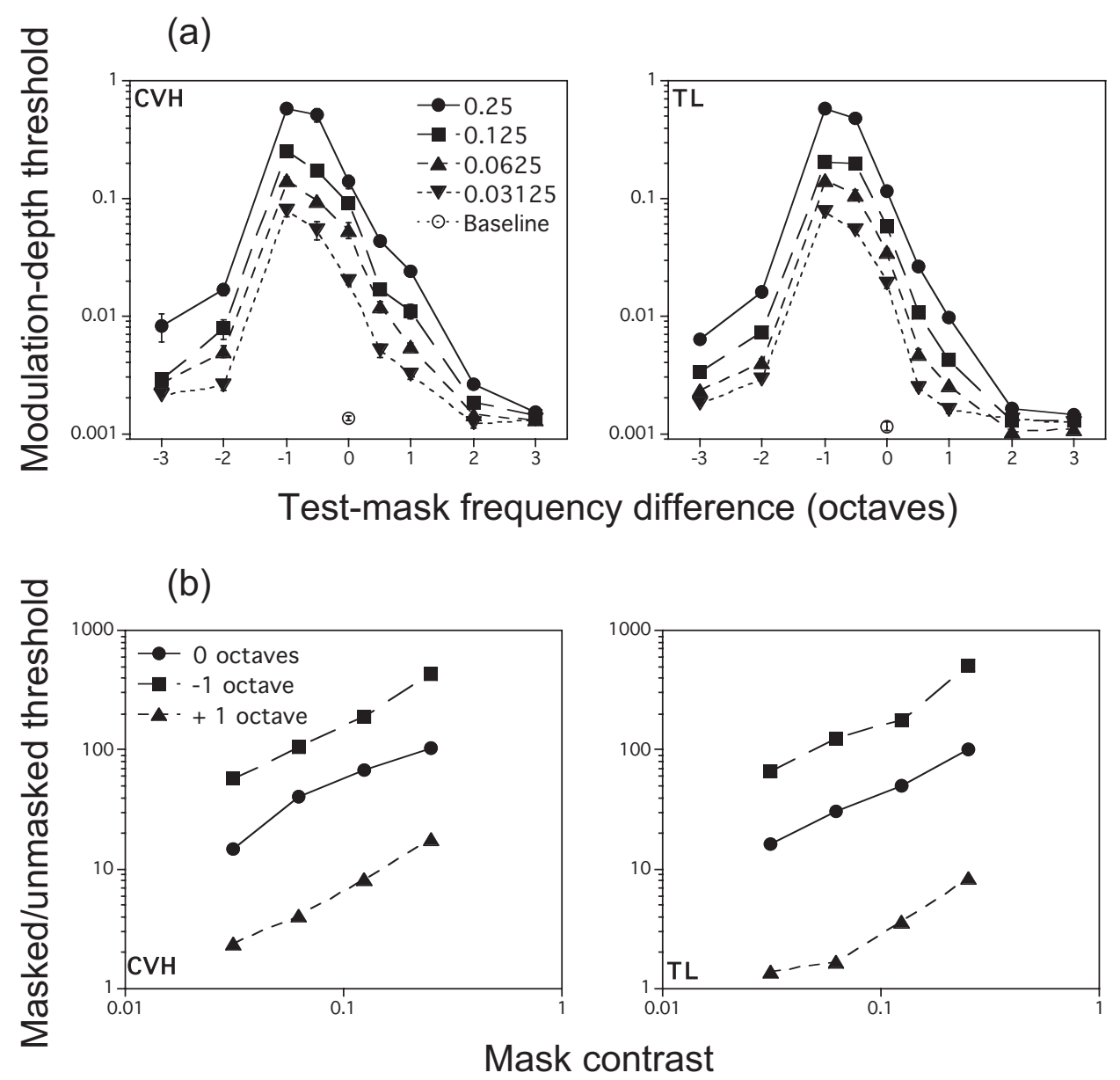

FIGURE 2. Masking for two observers $(\mathrm{CVH}, \mathrm{TL})$ measured at a test spatial frequency of $2 \mathrm{cyc} / \mathrm{deg}$. Masking effects were measured at four different mask contrasts: $0.03125,0.0625$, 0.125 , or 0.25 (Michelson). (a) Top: data for each observer were plotted as a function of mask separation (in octaves) from the test spatial frequency. Error bars, \pm 1 SEM. (b) Bottom mask-unmasked thresholds were plotted for each observer for test-mask frequency differences of $0,-1$, and +1 octaves, as a function of mask contrast.

a test frequency of $2 \mathrm{cyc} / \mathrm{deg}$, may indeed have reflected underlying sensitivity to the masks. Modulation sensitivity functions are band-pass in nature, peaking at $\sim 1 \mathrm{cyc} / \mathrm{deg}$ (corresponding to the mask positioned 1 octave below the $2 \mathrm{cyc} / \mathrm{deg}$ test pattern). Moreover, sensitivity was generally greater at frequencies below $2 \mathrm{cyc} / \mathrm{deg}$ than at frequencies above, in accordance with the general asymmetry in the masking functions at $2 \mathrm{cyc} / \mathrm{deg}$.

If the shapes of the masking functions do reflect differential sensitivity to the masks, then if the masks are presented at equal multiples of detection threshold, each mask should be of approximately equivalent contrast (detectability) to the visual system (assuming that the slopes of the contrast response functions are identical across spatial frequency). In this case, the asymmetry in the masking functions should disappear, and a "conventional" masking function should be observed where maximum masking occurs when the test and mask share the same frequency and decreases as the test-mask spatial frequency difference increases. To test this possibility, masking was measured at a test spatial frequency of $2 \mathrm{cyc} / \mathrm{deg}$, with each mask presented at an equal multiple of detection threshold. The mask pattern to which sensitivity was lowest was set to 0.25 Michelson contrast, and the contrasts of the masks at other spatial frequencies were adjusted so that they were at the same multiple above threshold. Maximum masking effects did not occur when the test and mask shared the same spatial (a)

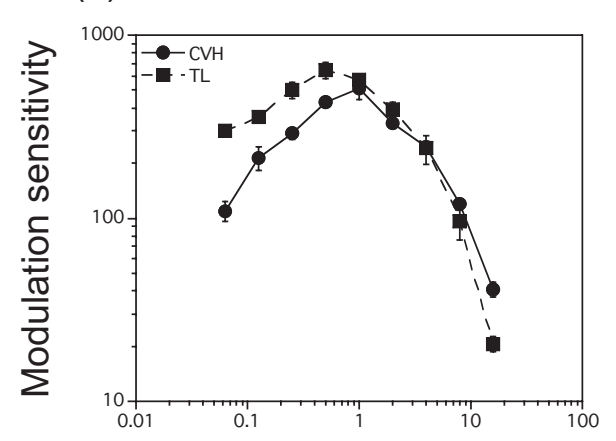

Mask spatial frequency (c/deg) (b)

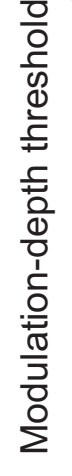

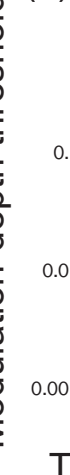

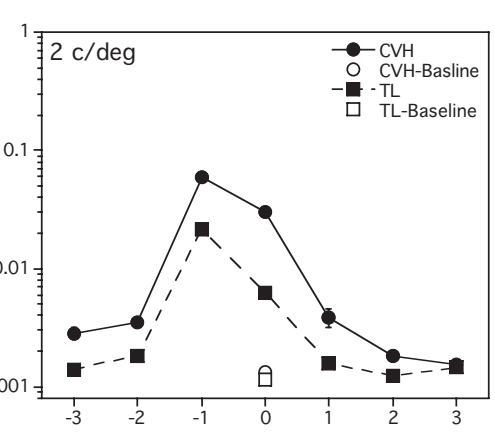

Test-mask frequency difference (octaves)
Figure 3. (a) Modulation sensitivity functions (reciprocal of modulation depth at detection threshold) for two observers (CVH, TL) for each jittering mask at spatial frequencies ranging from 0.0625 to $16 \mathrm{cyc} / \mathrm{deg}$. (b) Masking functions for two observers $(\mathrm{CVH}, \mathrm{TL})$ at a test spatial frequency of $2 \mathrm{cyc} / \mathrm{deg}$ where each mask pattern was presented at the same multiple of detection threshold. This threshold was 9.8 times that for observer $\mathrm{CVH}$ (contrast range: $0.02-0.25$ ) and 4.9 times for observer TL (contrast range: $0.008-0.25)$. The plots include baseline thresholds (open symbols) and masked thresholds (filled symbols). Error bars, \pm 1 SEM 
frequency but rather remained maximum when the mask was positioned at 1 octave below the test frequency (Fig. 3b).

As an additional control, we used a contrast-matching task in which observers made a subjective judgment about which of two patterns (a standard and a match) had the greater contrast. The standard pattern was a 2 -cyc/deg jittering grating, and the match pattern was a jittering grating that was $0.25,0.5,1,2,4$, 8 , or $16 \mathrm{cyc} / \mathrm{deg}$ (corresponding to each mask spatial frequency). A two-interval, forced-choice procedure was used in which the standard grating appeared in one interval, and the match grating appeared in the other (the grating that came first was randomized across trials with a probability of 0.5 ). The modulation depth of the standard grating was fixed at 0.25 and the modulation depth of the match grating varied. The observer's task was to judge which interval contained the grating with the highest contrast. The modulation depth at which the match grating was judged to have the same perceived contrast as the standard grating (the point of subjective equality) was estimated using an adaptive staircase technique in a manner analogous to that described in the Methods section.

Figure 4 a shows that the modulation depth at which the match grating was judged to have the same perceived contrast as the 0.25 contrast standard grating hovered at $\sim 0.25$ for match gratings that had low spatial frequencies $(\leq \sim 4 \mathrm{cyc} /$ deg). For higher spatial frequency match gratings, the modulation depth of the match grating that had the same perceived contrast as the standard grating rose rapidly (especially for observer $\mathrm{CVH}$ ).

Masking was then measured at a test spatial frequency of 2 cyc/deg with each mask presented at the modulation depth at which it had the same perceived contrast as the 2-cyc/deg standard grating. Once more, maximum threshold elevation did not occur when the test and mask shared the same spatial frequency but when the mask was 1 octave below the test frequency (Fig. 4b).

The masking functions produced by presenting the masks at equal multiples of threshold (Fig. 3b) exhibited less threshold elevation (masking) than those produced when masks were matched for perceived contrast (Fig. 4b), because in Figure $3 b$ the masks were of a much lower physical contrast than those in Figure $4 \mathrm{~b}$, thus resulting in less masking at each mask frequency. This result suggests that, although matching contrast by taking equal multiples of threshold is a commonly used method, it may not be the most appropriate technique for perceptually equating stimuli of different spatial frequencies. However, most important, in both cases maximum masking occurred when the mask was $\sim 1$ octave below the test frequency, and the masking functions do not differ markedly in terms of shape.

\section{Experiment 3: Speed}

In the two experiments outlined, although the test spatial frequency varied, the temporal frequency remained constant at $\sim 5 \mathrm{~Hz}$. As such, speed varied as a function of test spatial frequency. Specifically, speed decreased with increasing spatial frequency, from $20 \mathrm{deg} / \mathrm{sec}$ at a test spatial frequency of $0.25 \mathrm{cyc} / \mathrm{deg}$ to $1.25 \mathrm{deg} / \mathrm{sec}$ at a test spatial frequency of 4 cyc/deg. It is possible therefore that the off-frequency peak observed at higher test frequencies may be an effect of lower speeds, rather than spatial frequency, per se.

To investigate, we measured spatial-frequency-selective masking at test spatial frequencies of 0.25 and $2 \mathrm{cyc} / \mathrm{deg}$, both at a speeds of $5 \mathrm{deg} / \mathrm{sec}$, the assumption being that if the off-center peak was modulated by speed, then the peak at $\sim 1$ octave below the test spatial frequency should either become apparent for $0.25 \mathrm{cyc} / \mathrm{deg}$ or disappear for $2 \mathrm{cyc} / \mathrm{deg}$. As in experiment 1, mask contrast was fixed at 0.25 (Michelson). The findings are outlined in Figure 5 where it is evident that both masking functions remained comparable. At a test spatial frequency of $0.25 \mathrm{cyc} / \mathrm{deg}$, maximum masking occurred when the test and mask shared the same spatial frequency and decreased as the difference between the test and mask increased. At a test spatial frequency of $2 \mathrm{cyc} / \mathrm{deg}$ maximum masking effects remained at $\sim 1$ octave below the test spatial frequency. As such, it is clear that the off-center frequency peak is not simply an effect of speed.

\section{Experiment 4: Off-Frequency Looking}

Masking studies generally present a single mask in conjunction with the test pattern and vary the test-mask spatial frequency difference to provide an estimate of spatial frequency selectivity. However potential problems can arise from what is referred to as "off-frequency looking." Off-frequency looking occurs when an observer is able to center the channel used for detection on a point other than the test frequency, thereby maximizing the signal-to-noise ratio and increasing his or her ability to detect the test pattern. This concomitant lowering of the observer's thresholds can distort measurements of bandwidth and, most important in our case, channel shape. ${ }^{14,18}$ To ensure that the asymmetric shape of our masking functions was not due to off-frequency looking we remeasured the masking function at test spatial frequencies of 0.25 and $2 \mathrm{cyc} / \mathrm{deg}$ using two jittering masks (each 0.125 contrast), simultaneously superimposed above and below the test frequency. Results are shown in Figure 6. Figure 6a shows masking functions at a test spatial frequency of $0.25 \mathrm{cyc} / \mathrm{deg}$ where it is evident that maximum masking occurred when the masks and the test patterns had the same spatial frequency and decreased as the (a)

FIGURE 4. (a) Data for two observers showing the modulation depths of the match gratings at which they were perceived to be the same contrast as the standard grating, which was a 2-cyc/deg grating, the contrast of which was 0.25 . (b) Masking functions for two observers (CVH, TL) at a test spatial frequency of 2 cyc/deg, where each mask pattern was presented at the same perceived contrast. The plot includes baseline thresholds (open symbols) and masked thresholds (filled symbols). Error bars, \pm 1 SEM.

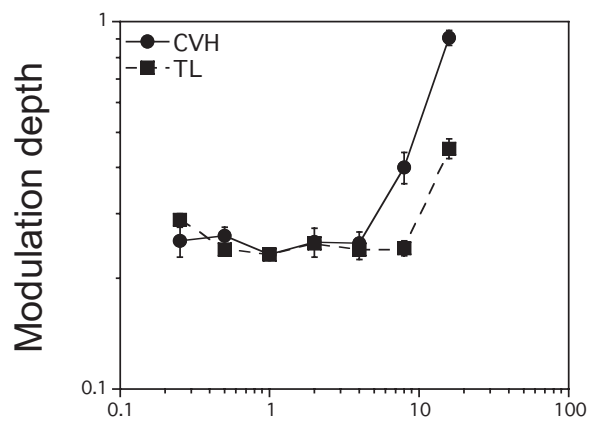

Match spatial frequency $(\mathrm{c} / \mathrm{deg})$ (b)

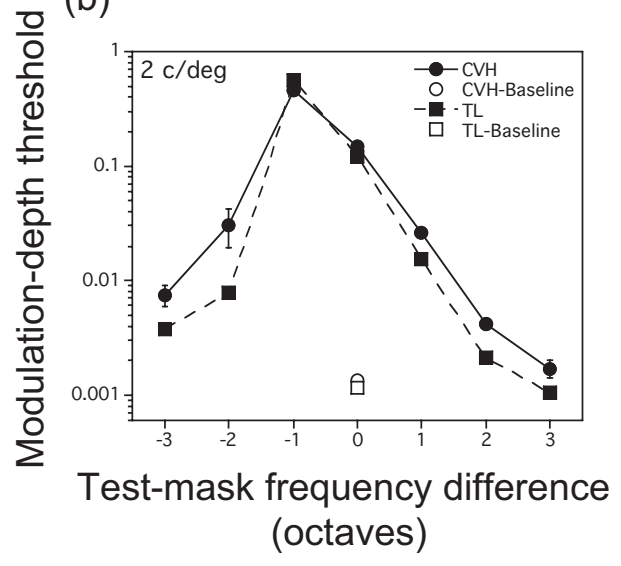


(a)

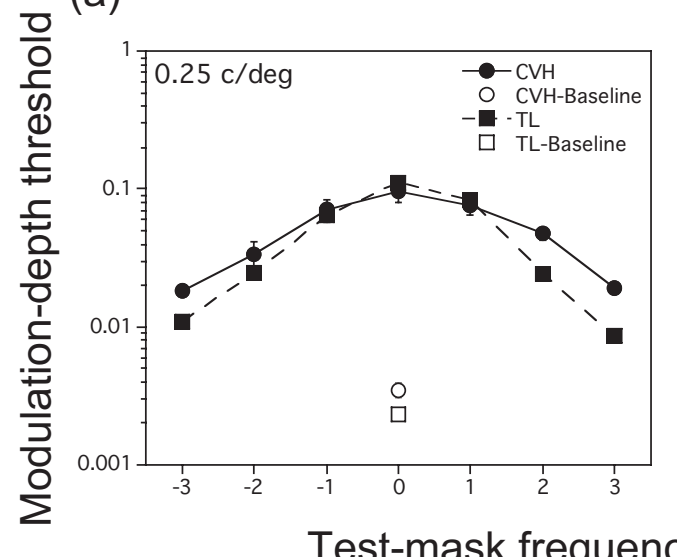

(b)

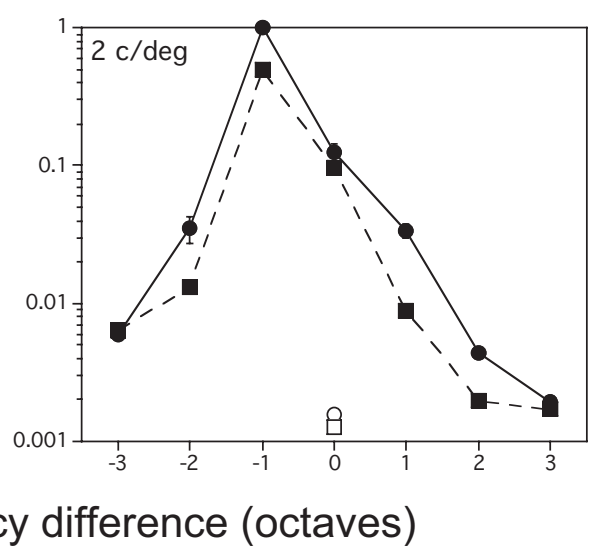

FIGURE 5. Masking functions for two observers (CVH, TL) at test spatial frequencies of (a) 0.25 and (b) 2 cyc/deg Both test patterns drifted at the same speed of $5 \mathrm{deg} / \mathrm{sec}$. Plots include baseline thresholds (open symbols) and masked threshold data (filled symbols). Error bars, \pm 1 SEM. two masks moved farther away in frequency from the test. Figure $6 \mathrm{~b}$ shows masking functions at a test spatial frequency of $2 \mathrm{cyc} / \mathrm{deg}$. In this instance, maximum masking occurred when the masks were positioned at $\sim 1$ octave from the test. That we obtained a standard masking function at $0.25 \mathrm{cyc} / \mathrm{deg}$ and an asymmetric masking function (with a peak at 1 octave from the test) at 2 cyc/deg clearly demonstrates that our masking results were not due to off-frequency looking.

\section{Experiment 5: Image Size and Number of Cycles}

Using the technique of visual masking, Anderson and Burr ${ }^{12}$ did not find any asymmetries in their spatial-frequency-selective tuning functions. However, unlike us, they kept 5 cycles of test grating on-screen in all conditions and varied viewing distance to change spatial frequency, whereas we kept viewing distance constant at $73 \mathrm{~cm}$ and varied the number of cycles presented on-screen from 5 cycles at a test frequency of 0.25 cyc/deg to $80 \mathrm{cycles}$ at $4 \mathrm{cyc} / \mathrm{deg}$. Therefore in experiment 5 , the masking function was remeasured at a test spatial frequency of 2 cyc/deg with five cycles of the test grating presented on-screen, resulting in an image that subtended $2.5^{\circ}$ both horizontally and vertically that was directly comparable to that used by Anderson and Burr ${ }^{12}$ at this test frequency. As in our initial masking experiment (experiment 1), a mask contrast of 0.25 was used. The results are shown in Figure 7 where the masking functions resemble those found previously, ${ }^{12}$ in that maximum masking effects occur when the test and mask share the same spatial frequency. Although the off-frequency peak disappears when the image is relatively small and only five cycles of grating are presented on-screen, the masking function is still somewhat asymmetric, in that the degree of threshold elevation is different at corresponding mask frequencies above and below the test frequency. For example, a mask at -3 octaves has a greater effect than a mask at +3 octaves.

\section{Discussion}

To our knowledge, the filter asymmetry revealed in the present study has not been reported elsewhere in the psychophysical motion literature. This lack of data may be due to several factors: (1) Investigators in previous masking studies have generally measured spatial frequency selectivity using static stimuli (with the exception of a few studies such as Anderson and Burr $^{12}$ ) whereas the effects presented herein may only apply to the operation of mechanisms sensitive to motion. (2) Those who have examined the spatial frequency tuning of motion masking have typically used rapid, but subjective, methods of threshold estimation due to the time-consuming nature of collecting large numbers of thresholds. Anderson and Burr, ${ }^{12}$ for example, used the method of adjustment in which observers turned a hand-held attenuator, until the drift direction of the test grating was just detectable. However, as previously mentioned, this method is subjective and does not necessarily guarantee an explicit judgment of motion, nor does it control the level of detectability to ensure equivalence across (a)

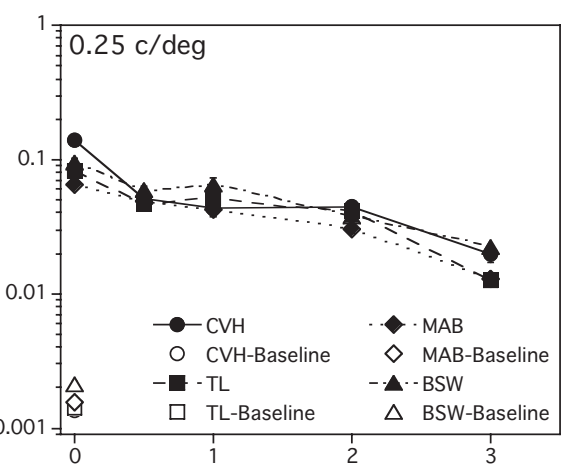

(b)

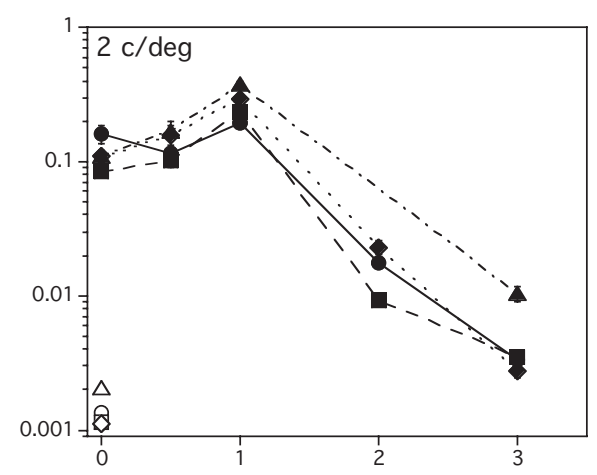

FIGURE 6. Masking functions for four observers (CVH, TL, MAB, BSW) at test spatial frequencies of (a) 0.25 and (b) $2 \mathrm{cyc} / \mathrm{deg}$ in the absence of off-frequency looking. Masking was measured for each test spatial frequency in the presence of two jittering masks, simultaneously positioned above and below the test frequency at testmask spatial frequency differences of 0.5 to 3 octaves. Plots include baseline thresholds (open symbols) and masked threshold data (filled symbols). Error bars, \pm 1 SEM. 
Figure 7. Masking functions for two observers $(\mathrm{CVH}, \mathrm{TL})$ at a test spatial frequency of $2 \mathrm{cyc} / \mathrm{deg}$. (a) Image size was $2.5^{\circ}$ and 5 cycles of test grating were presented on screen (as in Anderson and Burr ${ }^{12}$ ). Plots include baseline thresholds (open symbols) and masked threshold data (filled symbols). (b) Same as (a), except that data from an image size of $20^{\circ}$ (experiment 1) are shown (gray symbols) for comparison purposes. Error bars, \pm 1 SEM.

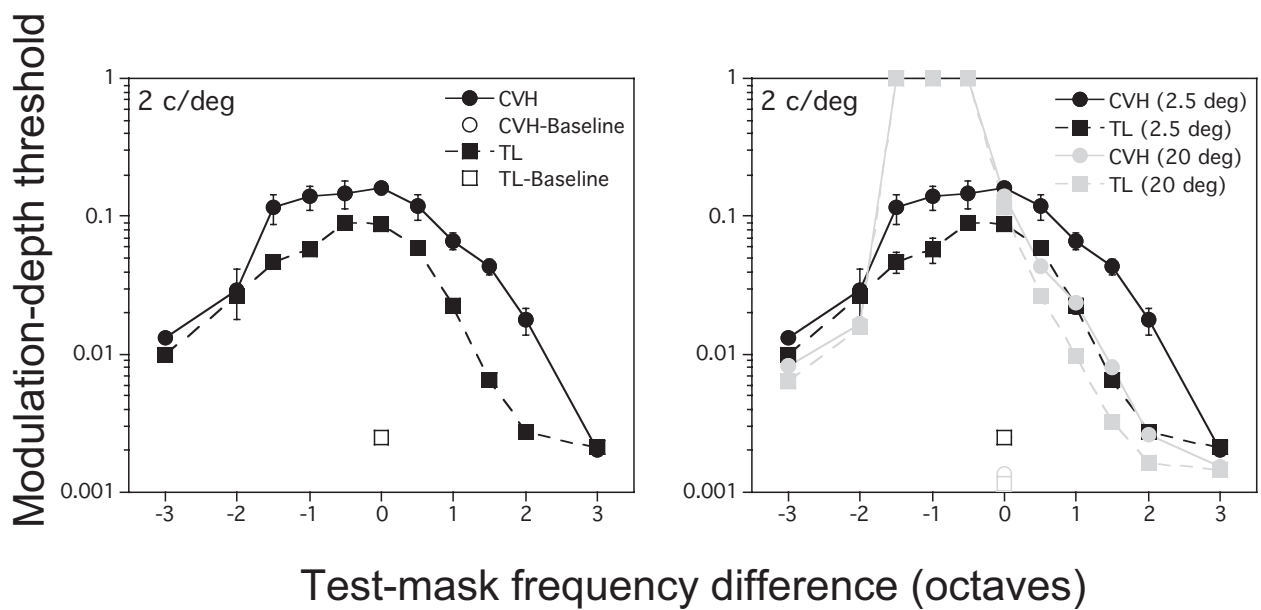

the conditions. (3) In addition, in these studies masking functions have been measured with a fixed mask contrast and have the effect of varying mask contrast on the corresponding tuning functions has not been measured. (4) In previous masking studies, researchers have used low test spatial frequencies and small (foveal) images and/or have presented relatively few cycles of grating on-screen. Under these conditions, we have shown that standard masking effects occur (i.e., peak masking when test and mask share the same frequency).

There is evidence of maximum masking effects at off-test frequency in the color domain. De Valois and Switkes, ${ }^{19}$ for example, measured masking effects using isoluminant chromatic, red-green grating patterns. Masking was measured at test frequencies of 1, 2, 4, and $8 \mathrm{cyc} / \mathrm{deg}$ with masks (20\% contrast) that were presented at a range of mask-test spatial frequency ratios. Results showed that only one subject demonstrated a peak masking level-calculated as $\log$ (contrast sensitivity in the presence of the mask)/(unmasked contrast sensitivity)when the test and mask shared the same spatial frequency. Three of four subjects did not show consistent centering of the masking effect. Rather, the greatest degree of masking occurred when the mask spatial frequency was twice that of the test. Moreover, this effect occurred regardless of the absolute frequency of the test or the mask patterns.

There also appear to be several other instances in the luminance domain in which maximum masking effects do not occur when test and mask share the same frequency. For example, Stromeyer and Julesz ${ }^{9}$ measured the detectability of sinusoidal luminance gratings under a variety of masking conditions and obtained standard masking functions while varying the spatial frequency of test gratings relative to various 1-octave wide bands of noise. Masking was also measured as a function of the width of a band of noise centered on the grating frequency. In this instance, masking increased as the noise widened from 0.75 to 1.5 octaves where it reached its peak, and further widening of the noise band led to a reduced masking level. Derrington and Henning ${ }^{20}$ have also found maximum masking effects at off-test frequency using luminancedefined grating patterns. They measured orientation and spatial frequency tuning by measuring the detectability of a range of vertically oriented test gratings (from 0.5 to $6 \mathrm{cyc} / \mathrm{deg}$ ) that were masked by two 3 -cyc/deg mask gratings oriented at $\pm 22^{\circ}$, $\pm 45^{\circ}$, and $\pm 67.5^{\circ}$ from vertical. The spatial frequency tuning of the masking effect reached its maximum at a test frequency of $\sim 2 \mathrm{cyc} / \mathrm{deg}, \sim 1 \mathrm{cyc} / \mathrm{deg}$ below the spatial frequency of the mask patterns.

A point noted by several authors ${ }^{21}$ and one worth reiterating is that although it has often been assumed that masking directly measures the tuning of a channel that corresponds to a particular frequency, it may be that in some cases what is actually being measured is interactions between channels (some form of between-channel inhibition). If this in indeed the case, then our findings suggest that for moving luminance patterns this inhibition is most evident when test spatial frequencies are $\geq 2 \mathrm{cyc} / \mathrm{deg}$ and increases with the number of spatial cycles of the grating presented on screen.

Although the dependence of this phenomenon on the number of spatial cycles has yet to be fully explored, inhibition between motion detectors maximally sensitive to different spatial frequencies has been proposed as a mechanism to explain other phenomena, such as misperception of the direction of compound gratings at suprathreshold stimulus levels. For example, Derrington and Henning ${ }^{22}$ reported that observers often misjudge the direction of a drifting 3-cyc/deg grating stimulus presentation is brief. They tentatively suggested that motion detectors responding to the $3-\mathrm{cyc} / \mathrm{deg}$ grating inhibit those tuned to the same direction, but lower spatial frequencies, that are stimulated by the directional noise resulting from the transient presentation of the $1 \mathrm{cyc} / \mathrm{deg}$ background grating. This inhibition could bias the perception of the net motion energy present in the stimulus in the direction opposite that of the drifting grating, resulting in errors in direction judgments.

Finally, we have recently shown ${ }^{23}$ effects comparable to those reported in the present masking study when we examined the spatial frequency tuning of the motion aftereffect (MAE). This finding is important for two reasons: (1) The observers who participated in our adaptation study were different from those who participated in our masking study, yet both groups exhibited qualitatively similar patterns of performance (i.e., asymmetric spatial frequency tuning to motion). Consequently, that there were different performers bolsters the general applicability of our results with regard to the expected prevalence of this phenomenon within the general population. ${ }^{24}$ (2) It is likely that our results reflect the underlying spatial frequency tuning of motion-sensitive mechanisms in the visual system rather than simply the mechanisms underlying the technique of visual masking.

As regards future work, we are currently exploring whether inhibition between motion detectors tuned to different frequencies, as suggested by Derrington and Henning, ${ }^{22}$ can account for the asymmetric masking functions we have reported in the present study and those we have found more recently using adaptation. ${ }^{23}$ superimposed on a 1-cyc/deg stationary background when the 


\section{Acknowledgments}

The authors thank Paul V. McGraw for assisting in the visual assessment of the observers and the two anonymous reviewers for helpful suggestions.

\section{References}

1. Campbell FW, Robson JG. Application of Fourier analysis to the visibility of gratings. J Physiol (Lond). 1968;197:551-566.

2. Blakemore C, Campbell FW. On the existence of neurons in the human visual system selectively sensitive to the orientation and size of retinal images. J Pbysiol (Lond). 1969;203:237-260.

3. Blakemore C, Nachmias J, Sutton P. The perceived spatial frequency shift: evidence for frequency-selective neurones in the human brain. J Pbysiol (Lond). 1970;210:727-750.

4. Blakemore C, Muncey JPJ, Ridley RM. Perceptual fading of a stabilised cortical image. Nature. 1971;233:204-205.

5. Blakemore C, Muncey JPJ, Ridley RM. Stimulus specificity in the human visual system. Vision Res. 1973;13:1915-1931.

6. Carter BE, Henning GB. Detection of gratings in narrow-band visual noise. J Pbysiol (Lond). 1971;219:355-365.

7. Graham N, Nachmias J. Detection of grating patterns containing two spatial frequencies: a comparison of single-channel and multiple-channels models. Vision Res. 1971;11:251-259.

8. Sachs MB, Nachimas J, Robson JG. Spatial-frequency channels in human vision. J Opt Soc Am. 1971;61:1176-1186.

9. Stromeyer CF, Julesz B. Spatial-frequency masking in vision: critical bands and the spread of masking. J Opt Soc Am. 1972;62:12211232.

10. Legge GE, Foley JM. Contrast masking in human vision. J Opt Soc Am. 1980;70:1458-1471.
11. Wilson HR, McFarlane DK, Phillips GC. Spatial frequency tuning of orientation selective units estimated with oblique masking. Vision Res. 1983;23:873-882.

12. Anderson SJ, Burr DC. Spatial and temporal selectivity of the human motion detection system. Vision Res. 1985;25:1147-1154.

13. Henning GB. Spatial-frequency tuning as a function of temporal frequency and stimulus motion. J Opt Soc Am A. 1988;5:1362-1373.

14. Hutchinson CV, Ledgeway T. Spatial frequency selective masking of first-order and second-order motion in the absence of off-frequency 'looking'. Vision Res. 2004;44:1499-1510.

15. Wright MJ. Spatiotemporal properties of gratings motion detection in the centre and periphery of the visual field. J Opt Soc Am A. 1987; $4: 1627-1633$.

16. Wetherill GB, Levitt H. Sequential estimation points on a psychometric function. Br J Math Stat Psychol. 1965;18:1-10.

17. Levitt H. Transformed up-down methods in psychoacoustics. $J$ Acoust Soc Am. 1971;49:467-477.

18. Losada MA, Mullan KT. Color and luminance spatial tuning estimated by noise masking in the absence of off-frequency looking. $J$ Opt Soc Am A. 1995;12:250-260.

19. De Valois KK, Switkes E. Simultaneous masking interactions between chromatic and luminance gratings. J Opt Soc Am. 1983;73: 11-18.

20. Derrington AM, Henning GB. Some observations on the masking effects of two-dimensional stimuli. Vision Res. 1989;29:241-246.

21. De Valois RL, DeValois KK. Spatial Vision. New York: Oxford University Press; 1990.

22. Derrington AM, Henning GB. Errors in the direction-of-motion discrimination with complex stimuli. Vision Res. 1987;27:61-75.

23. Ledgeway T, Hutchinson CV. Asymmetric spatial frequency tuning in the human visual motion system. Perception (suppl). 2007; 36(2):307.

24. Anderson AJ, Vingrys AJ. Small samples: does size matter? Invest Ophthalmol Vis Sci. 2001;42:1411-1413. 